Regular Article

\title{
Improving the Behavior of an Electro-Optic Modulator by Controlling Its Temperature
}

\author{
Dang Thanh Bui ${ }^{1}$, Lam Duy Nguyen ${ }^{2}$, Bernard Journet ${ }^{1}$ \\ ${ }^{1}$ SATIE, Institut d'Alembert, ENS Cachan, CNRS, PRES UniverSud, Cachan, France \\ ${ }^{2}$ Department of Physics, University of Pedagogy of Ho Chi Minh City, Vietnam
}

Correspondence: Bernard Journet, bernard.journet@satie.ens-cachan.fr

Manuscript communication: received 20 September 2010, accepted 16 January 2011

\begin{abstract}
The purpose of this paper is to present the improvement of the stability of an electro-optic modulator obtained from its temperature control. A digital system based on PSOC microcontroller and a small power amplifier has been developed. The behavior of the modulator can be observed by the way of its nonlinear properties. Thanks to the improved thermal stability, it is possible to reduce significantly the drift of the electro-optic modulator.
\end{abstract}

Keywords- Electro-optic modulator, linear behavior, second harmonic detection, temperature control, digital PID controller.

\section{INTRODUCTION}

Optical telecommunication systems at high data rate require external modulation. An electro-optic modulator $(\mathrm{EOM})$ is used for modulating the intensity of the laser beam according to the logical levels of the digital data. A good transmission is obtained in case of a wide open eye pattern, and the modulator must be biased for a linear operation. Unfortunately electro-optic modulators are not perfectly stable and the optimum bias point changes during the working time. In case of electrooptic polymer based devices the drift can be dramatic [1]. A first step in the complete control of the bias point is presented in this paper.

In this study a commercial electro-optic modulator made of lithium niobate material $\left(\mathrm{LiNbO}_{3}\right)$ has been used (ref. MX-LN-10 from Photline Company). This kind of modulator is much more stable than electrooptic polymer based devices. Nevertheless its thermal drift can be observed in a long-run time interval. Hence the efficiency of the control can be tested for this kind of modulator. The technique developed here can also be applied to the electro-optic polymer material modulators whose optical bias point highly depends on temperature $[2,3]$.

\section{The Electro-Optic Modulator Properties}

\subsection{Behavior of the EOM}

An electro-optic modulator is characterized by its static transfer function, which is the optical output power as a function of the voltage applied to the electrodes. The measurement is performed from a $7 \mathrm{~Hz}$ electrical triangle signal applied to the biasing electrode

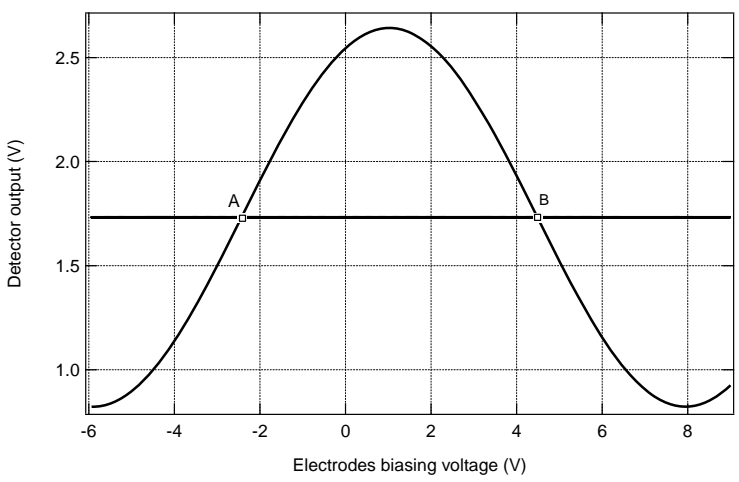

Figure 1. Transfer function of the electro-optic modulator. The average value of the signal is indicated by the horizontal line.

of the modulator. This frequency is low enough for considering that the transfer function corresponds to the static case. The resulting curve shown in Figure 1 presents the signal detected at the output of the modulator as a function of the slowly varying voltage applied to the DC electrode. The transfer function presents a sinusoidal behavior. The best linear behavior of the modulator is obtained at its half-transparency bias points such as A or B (see Figure 1).

\subsection{Determination of the Non-Linearity}

During the working time of the modulator there is a drift of the transfer function toward left or right on Figure 1. If the modulator has been initially biased with a constant DC voltage the effect of the drift will be a change of the bias point position on the transfer function and so a worst position according to the linear behavior of the modulator. The bias point is no longer at the optimal point. 


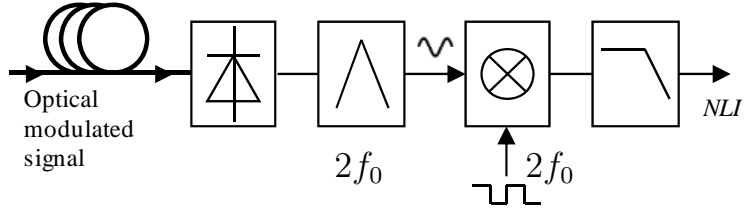

Figure 2. Block diagram of the measurement unit for estimating the non linearity of the modulator (NLI).

In order to check the linearity of the modulator a modulation of the optical beam going through the modulator is performed by the way of a sine voltage function, at a frequency $f_{0}$, applied to the DC electrode. If the modulator has a non linear behavior, high harmonic components will appear in the detected optical signal. For a bias point in the middle of the transfer function, harmonics may appear if the modulating signal is not small, but only with odd components. If the bias point is not in the middle of the transfer function the modulated signal is no longer symmetric and, in this case, there will be even components.

The quality criterion of the linearity of the modulator has to be based on the second harmonic component detection. But knowing the amplitude of the second harmonic of the modulated signal by itself is not enough for our method because there is no information about the direction of the bias point drift which is in fact given by the phase of the second harmonic signal. A simple solution for detecting the phase is to build a phase comparator based on a multiplier with a reference square signal at $2 f_{0}$ and a low pass filter giving the average value of the output of the multiplier which is an image of phase of the second harmonic signal.

The block diagram of the measurement unit is shown in Figure 2: the modulated signal is detected by a photodetector, the electrical signal goes through a bandpass filter centered at $2 f_{0}$, then there is the multiplier by " 0 " and "1" and the lowpass filter. At the output of the measurement unit, the signal is an algebraic estimation of the non linearity, it is called the Non-Linear-Indicator and noted NLI. This measurement unit is organized around a PSOC microcontroller from Cypress (ref. CY8C29466). The circuit is used for generating the modulating signal at $f_{0}=500 \mathrm{~Hz}$, the square signal at $2 f_{0}=1 \mathrm{kHz}$ and for performing the bandpass filtering which is based on switching capacitor technique. The quality factor of the filter is about $Q \approx 29.4$. The multiplier is a DG201 integrated circuit.

For determining the behavior of the modulator, its initial bias point is finely tuned in order to cancel the amplitude of the second harmonic, which is observed on a spectrum analyzer. A DC bias voltage is so applied to the modulator, and it is kept constant. Then the evolution of the second harmonic indicator NLI is recorded as shown in Figure 3. Without any temperature control there is clearly a drift of the transfer function leading to a non optimal bias point and a degradation of the non linearity. The next step is to control the temperature of the modulator, and to determine its influence on the

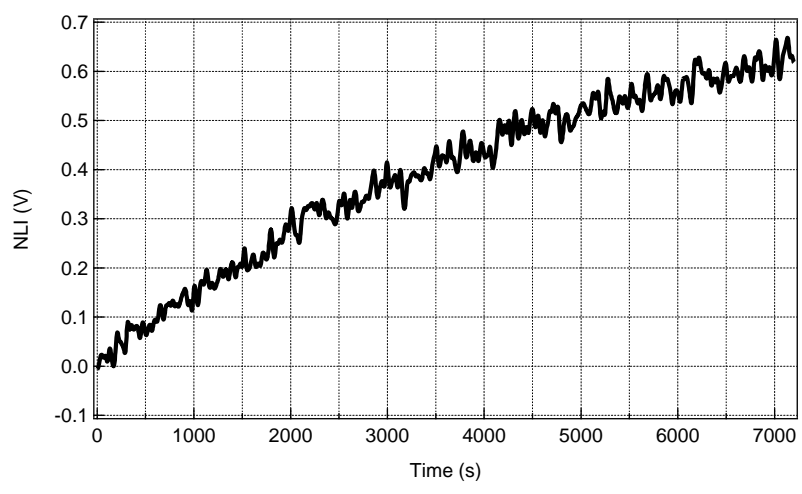

Figure 3. Evolution of the second harmonic component, without controlling the temperature of the modulator.

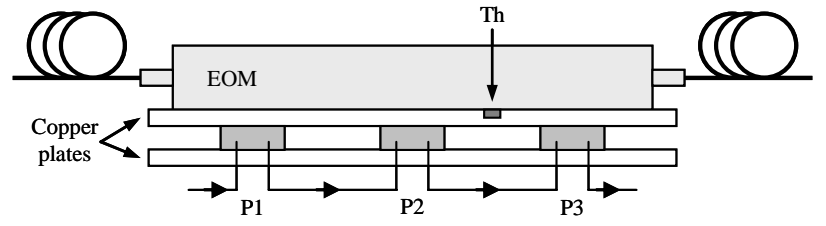

Figure 4. Electro-optic modulator (EOM), associated with a thermistor sensor (Th) and three Peltier modules (P1, P2 and P3).

behavior of the modulator.

\section{The Temperature Control Unit}

\subsection{Experimental Setup}

The experimental temperature control system is presented in Figure 4. The modulator is placed on a copper plate which stands on the upper side of three small Peltier modules connected in series and equally spaced along this plate. A second copper plate is placed on the bottom side of the Peltier modules. The temperature is tuned according to the sign and the intensity of the current going through these modules. A thermistor sensor is placed under the modulator between two of the Peltier modules; it is a part of a Wheatstone bridge followed by an instrumentation amplifier for measuring the temperature. The control unit board is designed around another similar PSOC microcontroller carrying a digital PID and achieving the data link with the computer. The electronic board performs the measurement of temperature, the digital control and gives an analog output driving a small power amplifier LM675T producing up to $\pm 1.5 \mathrm{~A}$ into the Peltier modules. The overall block diagram of the complete control system is presented in Figure 5.

\subsection{Implementation of the PID Controller}

In order to characterize the complete system, a current of $15 \mathrm{~mA}$ is applied to the Peltier modules. The evolution of the temperature as a function of time is shown in Figure 6. According to the step response, the system can be considered as being of first order with delay. By applying the Zigler-Nichols method $[4,5]$, it is possible to determine the parameters of the controller as presented in Table I. The equation of the 


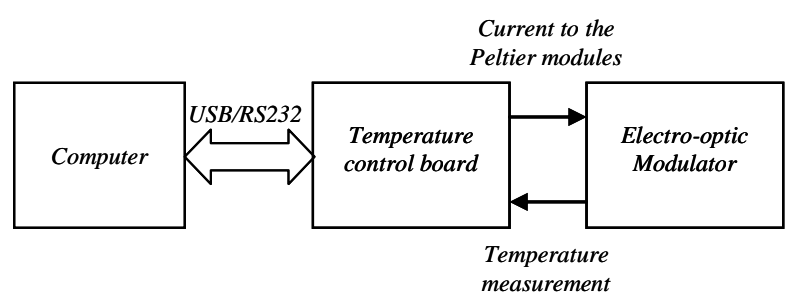

Figure 5. Overall block diagram of the electronic control unit.

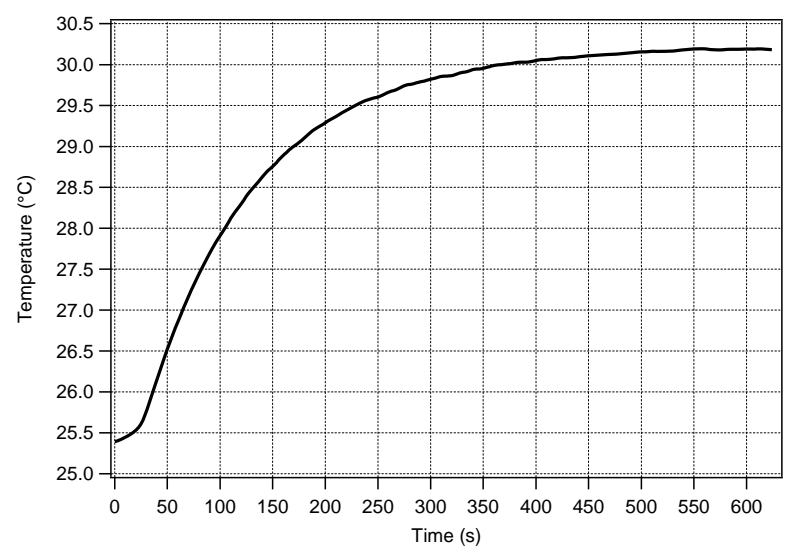

Figure 6. Evolution of the temperature of the EOM by applying a $15 \mathrm{~mA}$ driving current.

PID controller is given by [6]:

$$
u(t)=K_{p}\left[e(t)+\frac{1}{T_{I}} \int_{0}^{t} e(\tau) d \tau+T_{D} \frac{d e(t)}{d t}\right] .
$$

In case of digital technique, the PID is implemented in the microcontroller, as in (2) considering the sampling time $T_{0}$ which is $30 \mathrm{~ms}$ in this experiment and the discrete time $k=0,1, \ldots, t / T_{0}[6,7]$ :

$$
\begin{aligned}
u(k)= & K_{p}\left[e(k)+\frac{T_{0}}{T_{I}} \sum_{i=0}^{k} e(i-1)+\right. \\
& \left.\frac{T_{D}}{T_{0}}[e(k)-e(k-1)]\right] .
\end{aligned}
$$

\subsection{Temperature Control Efficiency}

A software has been developed in order to tune the temperature and the PID controller parameters. The working temperature of the modulator is recorded as a function of time for further processing. The Graphical User Interface (GUI) is shown in Figure 7. By using the PID parameters previously determined (see Table I), it is possible to observe the behavior of the system which is shown in Figure 8. The starting point was at room temperature of $17.4^{\circ} \mathrm{C}$, and the set point was $25^{\circ} \mathrm{C}$. The

Table I

PARAMETERS OF THE PID CONTROLler.

\begin{tabular}{|c|c|}
\hline $\begin{array}{c}\text { PID } \\
\text { parameters }\end{array}$ & Parameters value \\
\hline$K_{P}$ & 3.5 \\
\hline$T_{I}$ & 40 \\
\hline$T_{D}$ & 10 \\
\hline
\end{tabular}

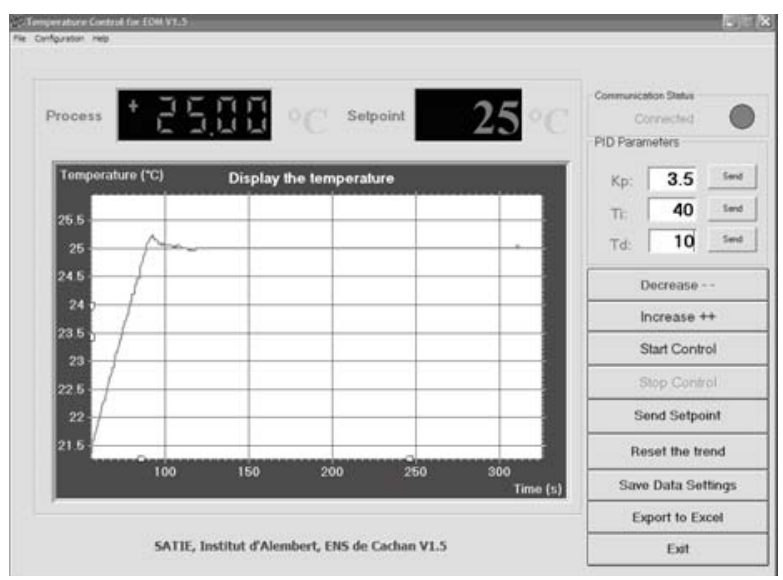

Figure 7. GUI of the control software.

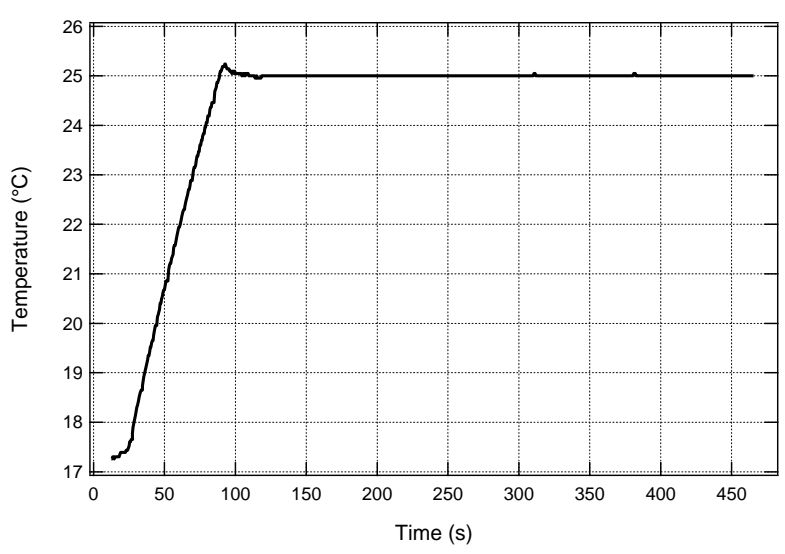

Figure 8. Evolution of the temperature with active PID Control.

observed overshoot is $0.25^{\circ} \mathrm{C}$, the settling time is $115 \mathrm{~s}$, and the steady-state error is $0.05^{\circ} \mathrm{C}$.

Such results are rather good for tuning and controlling the modulator temperature. The control unit should be able to follow the changing of the room temperature and to keep constant the modulator temperature.

\section{Temperature EfFect Investigations}

\subsection{Influence of the temperature on the EOM Bias Point}

The first experiment has been conducted in order to prove the influence of the temperature on the optimal modulator bias point. Some temperature changes by steps of $5{ }^{\circ} \mathrm{C}$ have been applied to the modulator and the corresponding non linearity has been recorded as shown in Figure 9. Temperature and bias point drift are clearly correlated. But when the temperature is decreased by $5^{\circ} \mathrm{C}$ to $25^{\circ} \mathrm{C}$ for the second time, the non linear indicator NLI does not return to the previous level. There are obviously other physical effects leading to the natural drift of the transfer function. 

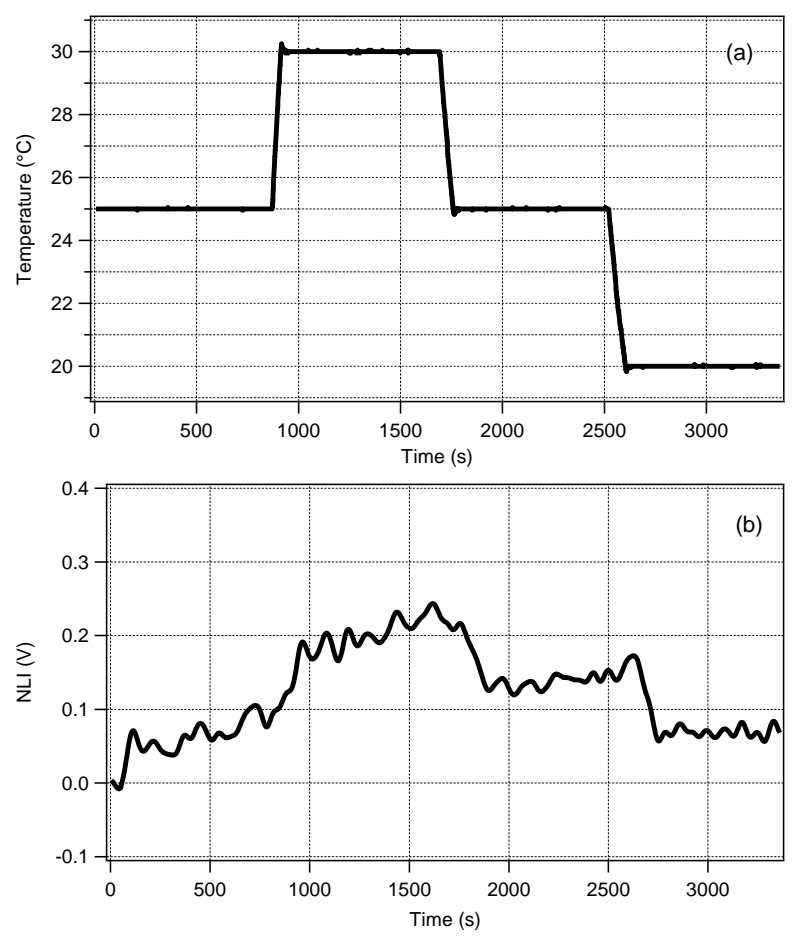

Figure 9. Temperature variations applied to the modulator by using the control unit (a) and the corresponding non-linearity criterion NLI (b).
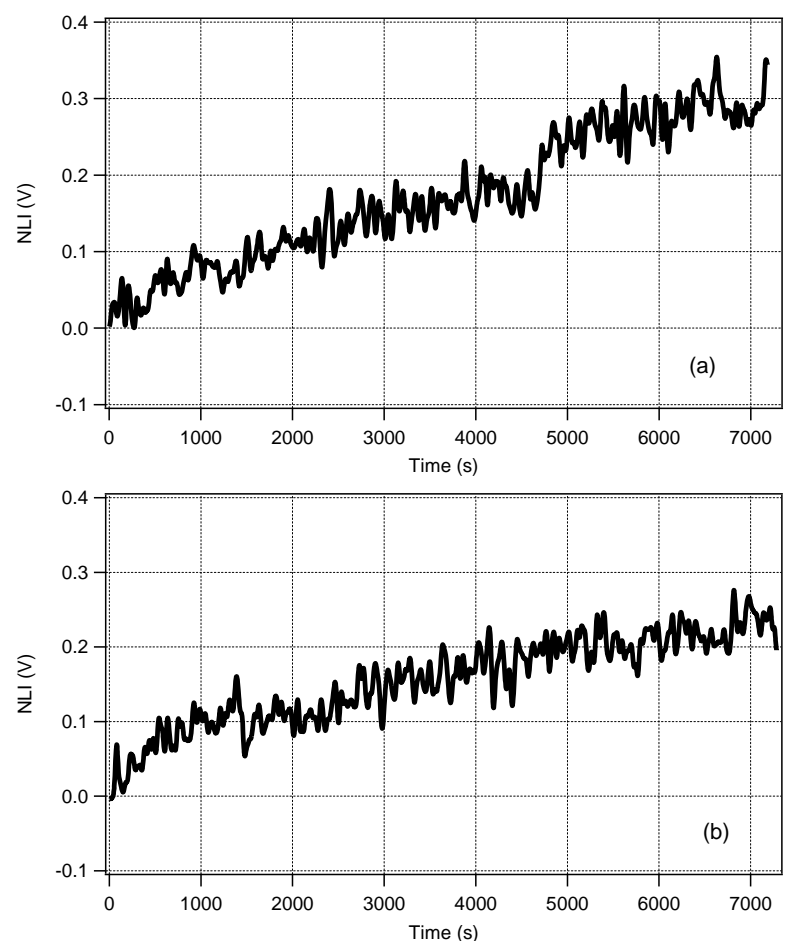

Figure 10. Evolution of the non-linearity without temperature control (a), and by controlling the temperature (b).

\subsection{Behavior of the EOM with Temperature Control}

The second experiment is a study of the efficiency of the temperature control unit on the modulator behavior. The drift of the bias point is presented without controlling the temperature (see Figure 10a) and in case of temperature control (see Figure 10b). The measure- ments have been conducted on the same day with quite similar room conditions. For an experimental duration of $7000 \mathrm{~s}$ and from the average slopes of the curves, it can be found that the NLI value has been reduced by $25 \%$ by stabilizing the temperature.

It can be supposed that the reduction of the drift depends on the working conditions and, among them, the evolution of the room temperature. Comparing Figures 3 and 10a we can see that different drift values can be obtained, and so we can suppose that the temperature control effects could be different. Further investigations have to be performed.

Obviously the drift of the bias point depends not only on the temperature parameter but also on other physical effects. A complete control is required for a better compensation of the modulator transfer function drift effects.

\section{Conclusion}

In this paper it has been shown that controlling the temperature of an electro-optic modulator improves its behavior: the effects of the transfer function drift in terms of non linearity can be reduced at least by $25 \%$. The work has been conducted with a lithium niobate modulator but it can be applied to modulators made of electro-optic polymer that are known as less stable. As there are different physical effects leading to the drift, direct control of the bias point should be achieved simultaneously with the temperature control.

\section{ACKNOWLEDGMents}

The authors would like to thank Mr. André Clouqueur and Dr. Chi Thanh Nguyen (LPQM, ENS Cachan) for the fruitful discussion we had together about the method used here for determining the non-linearity of the electro-optic modulator. The authors would also like to thank the EADS Foundation for supporting their research activities in the field of "Photonic devices based on electro-optic polymer materials" and the corresponding applications. This work has also been supported by Institut d'Alembert at ENS Cachan.

\section{REFERENCES}

[1] Y. Shi, W. Wang, W. Lin, D. J. Olson, and J. H. Bechtel, "Long-term stable direct current bias operation in electrooptic polymer modulators with an electrically compatible multilayer structure," Applied Physics Letters, vol. 71, no. 16, pp. 2236-2238, Oct. 1997.

[2] S. Park, J. J. Ju, J. Y. Do, S. K. Park, and M.-H. Lee, "Thermal stability enhancement of electrooptic polymer modulator," IEEE Photonics Technology Letters, vol. 16, no. 1, pp. 93-95, Jan. 2004.

[3] H. L. S. M. Aillerie, N. Théofanous, "Thermo-optic effects in an electro-optic modulation system," in Proc. New Achievements in Materials and Environmental Sciences (NAMES), 3rd France-Russia Seminar, Metz, France, 2007, pp. 87-91.

[4] D. Lequesne, Régulation P.I.D. : Analogique - Numŕique Floue. Lavoisier, 2006. 
[5] F. H. Raven, Automatic Control Engineering. McGraw-Hill, 1995.

[6] Cypress, "Sensing - universal pid-thermoregulator," Cypress Semiconductor Corporation, Tech. Rep. AN2208, 2005.

[7] M. M. Ashry, Z. Z. Kamalova, and T. V. Breikin, “Tuning of digital PID controller parameters using local optimal control," in Proc. 16th Mediterranean Conf. Control and Automation, Ajaccio, France, June 2008, pp. 587-592.

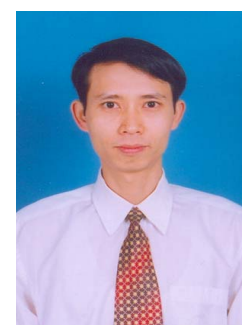

Dang Thanh Bui was born in 1976. He received the Master degree in Instrumentation and Control from Hanoi University of Science and Technology (HUST), Vietnam, in 2001. From 2001 to 2007, he was lecturer at the department of Instrumentation and Industrial Informatics of HUST. He is currently a $\mathrm{PhD}$ student in SATIE laboratory, d'Alembert Institute, ENS Cachan, France. His main research interest are control system design, microcontroller and DSP controller programming.

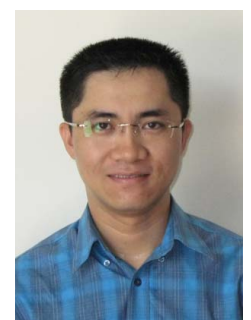

Lam Duy Nguyen was born in Ninh Thuan, Viet Nam, in 1980. He received his bachelor degree in physics from the Pedagogy University of Ho Chi Minh City (Vietnam) and his master degree from the Paris XI Orsay University (France) about Components and Antennas for Telecommunications in 2002 and 2006, respectively. He has got his PhD degree from the Ecole Normale Supérieure de Cachan (France), in June 2010. His thesis research was on the applications of optoelectronic oscillators. He is currently Assistant Professor at Pedagogy University of Ho chi Minh City.

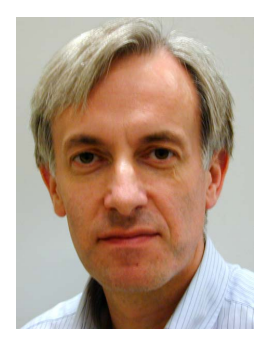

Bernard Journet was born in 1956 and received his Doctor degree in 1983 from the Paris VI Pierre et Marie Curie University about long period seismological waves, data acquisition and processing. He joined the ENS Cachan in 1989 as an Associate Professor, and is teaching physics, electronic and optoelectronic. He has been working on laser range finders, gas analysis and secured optical telecommunications. He has in charge the microwave photonic research team at SATIE laboratory ( $d$ 'Alembert Institute) developing applications of the optoelectronic oscillator. 